\title{
Implanted devices: biocompatibility, infection and tissue engineering
}

\author{
Werner Zimmerli
}

Received: 13 January 2011 / Accepted: 14 February 2011 / Published online: 21 May 2011

(C) Springer-Verlag 2011

In modern medicine, almost any part of the body can be replaced by an artificial implanted device. Due to a growing number of people with degenerative diseases, and thanks to the evolving technology of implants, the use of implanted medical devices is steadily increasing. Since more than 50 years, biomaterials have been developed for the use in human medicine. The first breakthrough in the research field of artificial organs was the replacement of degenerated joints in the 1960s. In the meantime, the knowledge about biomaterials has immensely evolved. Of note, the term 'biomaterial' is not only used for biological substances, but also for synthetic materials in implants.

Organs with complex function such as lung, liver, pancreas, heart and kidney cannot be permanently replaced by totally implanted artificial devices, but only by organ transplantation [1]. If in patients with terminal heart failure no organ is available, the failed heart can be replaced by a left ventricular assist device. However, this is not a definite solution, because not the whole device can be implanted in the body. Part of the device (electrical leads and battery) is still outside of the body [2]. Due to the connection between the intracorporal and extracorporal part of the device, infection and inadequate clotting remain as major problems. Therefore, the total artificial heart cannot yet permanently replace the damaged organ, but only bridge the time until a donor organ is available [2].

In contrast to the mentioned organs, other parts of the human body, such as joints, blood vessels or crucial components of organs (e.g. heart valves) can be replaced

W. Zimmerli $(\bowtie)$

Basel University Medical Clinic Liestal,

Kantonsspital, Rheinstrasse 26,

CH-4410 Liestal, Switzerland

e-mail: Werner.zimmerli@unibas.ch with implanted devices made from biomaterials of different complexity. Whereas, transplantation is hampered by shortage of organs, implantation of artificial devices exclusively depends on technology, surgical skills and financial means. Patients receiving organs commonly have to be immunosuppressed in order to avoid rejection. Implanted devices, however, are, in general, perfectly accepted by the host, whether they are made of devitalized organic material or of various synthetic materials [3]. The main drawback of transplanted organs is apparently the need for prolonged (stem cell transplantation) or even lifelong (solid organ transplantation) immunosuppression. The disadvantages of implanted artificial devices are less obvious. They can be summarized as increased susceptibility to perioperative exogenous infection, life-long risk for haematogenous implant-associated infection, suboptimal function leading to inflammation (e.g. wear particles), inadequate clotting (e.g. vascular devices) or haemolysis (e.g. mechanical valves especially in case of paravalvular leakage). Part of these problems will be covered in this special issue of Seminars in Immunopathology.

The most frequently used implants are in the field of orthopaedic surgery, namely internal fixation devices after bone fracture and artificial joints for primary osteoarthritis. During a 15-year period, the number of primary hip arthroplasty increased 1.6-fold to 226,811 , and the one of primary knee arthroplasty 2.8 -fold to 459,594 in the USA alone [4]. Therefore, the outcome after implantation of an orthopaedic device is not only important for the individual patient, but also has an economic impact.

This special issue of Seminars in Immunopathology focuses on the interaction between the host, its immune system and the artificial organ. It is indeed intriguing to follow these interactions between own and foreign. In view of the important role of orthopaedic implants, several 
orthopaedic aspects are presented in different contributions in this issue of Seminars in Immunopathology.

The review by Anderson and McNally [5] clearly shows that foreign devices are not inert, but that the host reacts with a well-defined part of the immune system. The extent of this host reaction can be graduated as biocompatibility. Innate as well as adapted cell interactions occur at sites of implanted devices. In addition, even systemic effects can be observed with certain devices. The authors discuss the example of a systemic effect of the left ventricular assist device on host defence. In patients with a left assist device, circulating CD4+ lymphocytes decrease by apoptosis. Furthermore, B-lymphocyte activation interferes with later donor heart acceptance. This illustrates that biocompatibility does not only relate to the behaviour of biomaterial at the site of implantation, but to a systemic reaction within the whole body also.

In the contribution of Binnebösel et al. [6], the reaction of the organism to prosthetic mesh grafts in abdominal surgery is presented. For mechanical reasons, meshes, of chemically different materials, are mainly used to repair hernias. The authors discuss the biocompatibility of specific chemical and physical characteristics of mesh grafts.

In orthopaedic surgery, mechanical stability of bone is crucial for an optimal functional result. Peter E. Ochsner [7] describes in his review the mechanisms that relate to osteointegration. Not only the type of material, but also the area of implantation is crucial for the process of osteointegration. In addition, shape, elasticity and surface structure of the implant are important factors defining the result regarding biocompatibility and biomechanics.

The friction of material after total joint replacement results in different amounts of wear particles. The review by Catelas et al. [8] highlights the effect of wear particles on the host. Accumulation and phagocytosis of such particles induce liberation of pro-inflammatory cytokines, a process which may lead to device loosening at the site of implantation. This biological response is dependent on chemical and physical characteristics of the particle.

After implantation of a device, osteointegration is beneficial for the host. However, heterotopic ossification is a feared complication occurring after various orthopaedic interventions. Zeckey et al. [9] show in their review that only little is known about the host factors inducing heterotopic ossification. They review pathophysiologic factors, risk factors, prevention and treatment options.

Since almost three decades, there is a debate regarding the association of silicone breast implants with connective tissue diseases. After an initial observation in Japan, a causal correlation between autoimmune disorders and silicone breast implants has been repeatedly claimed. In their contribution, Lipworth et al. [10] review the literature in the field, and conclude that there is no causal connection between silicone breast implants and connective tissue diseases.

Implantation of a foreign device in the host is not rarely complicated by infection, not only exogenously at time of surgery, but life-long by haematogenous seeding. Factors leading to this increased susceptibility to implant-associated infection are summarized in the review of Zimmerli and Sendi [11]. The main problem is the locally acquired inefficient host defence around foreign devices. Many of the reviews in this issue show limitations and unwanted effects of implanted devices. In the future, it is conceivable that tissue engineering overcomes these drawbacks [12]. Weber et al. [13] present in their review that this technology is no longer pure science fiction. Cardiovascular tissue engineering has already been shown to work in animals. This technology is promising and may replace the need of artificial devices in the future, at least in some fields of medicine.

\section{References}

1. Papafragkakis H, Martin P, Akalin E (2010) Combined liver and kidney transplantation. Curr Opin Organ Transplant 15:263268

2. Frazier OH, Gemmato C, Myers TJ, Gregoric ID, Radovancevic B, Loyalka P, Kar B (2007) Initial clinical experience with the HeartMate II axial-flow left ventricular assist device. Tex Heart Inst J 34:275-281

3. Oxenham H, Bloomfield P, Wheatley DJ, Lee RJ, Cunningham J, Prescott RJ, Miller HC (2003) Twenty year comparison of a Bjork-Shiley mechanical heart valve with porcine bioprostheses. Heart 89:715-721

4. Kurtz SM, Lau E, Schmier J, Ong KL, Zhao K, Parvizi J (2008) Infection burden for hip and knee arthroplasty in the United States. J Arthroplasty 23:984-991

5. Anderson JM, McNally A (2011) Biocompatibility of implants: lymphocyte/macrophage interactions. Semin Immunopathol (in press)

6. Binnebösel M, von Trotha KT, Jansen PL, Conze J, Neumann UP, Junge K (2011) Biocompatibility of prosthetic meshes in abdominal surgery. Semin Immunopathol (in press)

7. Ochsner PE (2011) Osteointegration of orthopedic implant material. Semin Immunopathol (this issue)

8. Catelas I, Utzschneider S, Wimmer MA (2011) Tissue reaction to metal and polyethylene wear particles: characteristics and biological effects. Semin Immunopathol (in press)

9. Zeckey C, Hildebrand F, Frink M, Krettek C (2011) Heterotopic ossifications following implant surgery-epidemiology, therapeutical approaches and current concepts. Semin Immunopathol (in press)

10. Lipworth L, Holmich LR, McLaughlin JK (2011) Silicone breast implants and connective tissue disease: no association. Semin Immunopathol (in press)

11. Zimmerli W, Sendi P (2011) Pathogenesis of implant-associated infection: the role of the host. Semin Immunopathol (in press)

12. Bhatia SK (2010) Tissue engineering for clinical applications. Biotechnol J 5:1309-1323

13. Weber B, Emmert MY, Schönauer R, Brokopp C, Baumgartner L, Hoerstrup SP (2011) Tissue engineering on matrix: Future of autologous tissue replacement. Semin Immunopathol (in press) 